\title{
N-terminal fragments of Huntingtin longer than residue 170 form visible aggregates independently to polyglutamine expansion.
}

Moore Z. Chen ${ }^{\mathrm{a}}$, Sue-Ann Mok ${ }^{\mathrm{b}}$, Angelique R Ormsby ${ }^{\mathrm{a}}$, Paul J. Muchowski ${ }^{\mathrm{c}}$, and Danny M. Hatters $^{\text {a* }}$

Running title: Aggregation of Htt fragments

* Corresponding author: Danny M. Hatters dhatters@ unimelb.edu.au. Phone: +61 383442530.

${ }^{a}$ Department of Biochemistry and Molecular Biology and Bio21 Molecular Science and Biotechnology Institute. The University of Melbourne, VIC 3010, Australia

${ }^{\mathrm{b}}$ Department of Neurology, University of California San Francisco, San Francisco, CA, 94158

${ }^{c}$ KynuRex. 634 Los Palmos Drive. San Francisco, CA 94127

Keywords: Aggregation, inclusion, polyglutamine, live cell imaging, Huntington's Disease, proteolytic fragments.

\begin{abstract}
Background: A hallmark of Huntington's disease is the progressive aggregation of full length and N-terminal fragments of polyglutamine (polyQ)-expanded Huntingtin ( $\mathrm{Htt}$ ) into intracellular inclusions. The production of $\mathrm{N}$-terminal fragments appears important for enabling pathology and aggregation; and hence the direct expression of a variety of $\mathrm{N}$-terminal fragments are commonly used to model HD in animal and cellular models.
\end{abstract}

Objective: It remains unclear how the length of the N-terminal fragments relates to polyQ mediated aggregation. We investigated the fundamental intracellular aggregation process of eight different-length N-terminal fragments of Htt in both short (25Q) and long polyQ (97Q).

Methods: N-terminal fragments were fused to fluorescent proteins and transiently expressed in mammalian cell culture models. These included the classic exon 1 fragment ( 90 amino acids) and longer forms of 105, 117, 171, 513, 536, 552, and 586 amino acids based on wild-type Htt (of 23Q) sequence length nomenclature.

Results: N-terminal fragments of less than 171 amino acids only formed inclusions in polyQexpanded form. By contrast the longer fragments formed inclusions irrespective of Q-length, with Q-length playing a negligible role in extent of aggregation. The inclusions could be classified into 3 distinct morphological categories. One type (Type A) was universally associated with polyQ expansions whereas the other two types (Types B and C) formed independently of polyQ length expansion.

Conclusions: PolyQ-expansion was only required for fragments of less than 171 amino acids to aggregate. Longer fragments aggregated predominately through a non-polyQ mechanism, involving at least one, and probably more distinct clustering mechanisms. 


\section{BACKGROUND}

CAG-trinucleotide expansion mutations, which encodes a polyglutamine stretch within exon 1 of the HTT gene beyond 36 repeats causes Huntington's disease (HD) [1]. A key feature of HD pathology are intracellular inclusion bodies comprising $\mathrm{N}$-terminal fragments of mutant Huntingtin (Htt) that encompass the polyQ segment [2]. The exogenous expression of exon 1 in polyQ-expanded form is sufficient to recapitulate neurological defects and aggregation pathology resembling some features of HD in rodent and primate models [3-5]. Because of this, the Htt exon 1 fragment, which comprises 90 amino acids1, has been extensively used to model the molecular mechanisms of HD.

The fragmentation process of Htt, and possibly direct production of truncated aberrantly spliced translation fragments, seems to be critical to mediating pathology [6-8]. Htt forms an array of different size cleavage products, putatively by caspase, calpain and other protease activity that precede pathogenesis $[9,10]$. The transgenic expression of the shorter Htt fragments generally confers greater toxicity than longer lengths, as demonstrated recently in a systematic comparative study in Drosophila [11] as well as in mouse models where shorter fragments typically invoke a more severe phenotype [12]. Mouse models that suppress generation of the N-terminal cleavage products by mutation of putative caspase proteolytic cleavage sites in Htt also lead to delayed pathologies [13].

Some of the key fragmentation steps of Htt or commonly used fragment models are summarized as follows. Caspase family (and possibly other [14]) protease cleavage products have been suggested to occur at aspartate residues 513, 552, and 586 [13, 15-17]. Calpain cleavage products have also been shown to occur cleaving at residues 469 and 536 and appear important for mediating aggregation and toxicity in cell culture $[7,17]$. Fragments from human brain inclusions, designated as cp-A and cp-B are of between 104-114 and 146-216 respectively [18-21]. Mouse models expressing the 171 fragment have been made that like Htt exon 1 can model HD pathogenesis [22].

During our studies on different proteolytic lengths we found that the aggregation pattern in cultured mammalian cells was not necessarily related to the length of polyQ. Given the importance of aggregation as a marker of pathology and its sometimes controversial role in relating to toxicity [23], we sought to systematic define the relationship between fragment length and polyglutamine length on aggregation patterns. Our findings described here indicate aggregation into visible inclusions is a complex pleiotropic-like phenomenon that in some contexts is poorly related to polyQ length. These results highlight the importance of understanding the molecular bases of aggregation phenomena observed morphologically in cellular contexts.

\section{MATERIALS AND METHODS}

\section{Plasmid vector construction}

The cDNA of full length human Htt (in 25Q form) was amplified into the fragment lengths shown in Fig. 1 by PCR; and then cloned into pEGFP-N1 via the SmaI and BamHI sites. The resultant constructs expressed EGFP as C-terminal fusions to the Htt fragments. The 97Q forms were made by replacing the 25Q cDNA with the corresponding cDNA sequence from a previously described pTIREX plasmid encoding human Htt exon 1 with 97Q [24] via internal restriction sites (EcoN1 and SacII). All constructs were sequenced for verification. N90 plasmids fused to mCherry were

1. Sequence numbering using Htt with 23Q length. This numbering convention is used hereon. 
prepared in the pGW1 vector as previously described, and the vectors for this were kindly provided by Steven Finkbeiner [25].

\section{Cell culture}

African green monkey kidney fibroblast-like cell line COS1 and human epithelial kidney cell line AD293 were cultured in complete DMEM (Invitrogen) media supplemented with $10 \%$ (v/v) fetal calf serum (FCS), $1 \mathrm{mM}$ L-glutamine, and $200 \mu \mathrm{g} / \mathrm{ml}$ penicillin/streptomycin. Mouse neuroblastoma cell line Neuro-2a was cultured in complete OptiMEM (Life Technologies)) media supplemented with $10 \%$ (v/v) FCS, $1 \mathrm{mM}$ L-glutamine and $100 \mu \mathrm{g} / \mathrm{ml}$ penicillin/streptomycin. Cells were cultured at $37{ }^{\circ} \mathrm{C}$ in a humidified incubator with $5 \%$ atmospheric $\mathrm{CO}_{2}$. Cells were passaged every two days, with COS1 and Neuro2a passaged at 1/4 dilution and AD293 at 1/8 dilution.

\section{Transfections}

For flow cytometry, $1.4 \times 10^{5} \operatorname{COS} 1$ cells were plated per well on 24 -well plates. The next day cells were transfected with Lipofectamine 2000 (Invitrogen) reagent containing $640 \mathrm{ng} \mathrm{Htt}$ fragment-EGFP vector and $160 \mathrm{ng}$ mCherry vector. For confocal microscopy, $9 \times 10^{4} \mathrm{Neuro} 2 \mathrm{a}$ cells or $7 \times 10^{4}$ COS1 or AD293 cells were plated per well on 8 -well $\mu$-slides (Ibidi). For the cotransfection experiment of N90 with N586, COS1 cells were transfected with $100 \mathrm{ng}$ N90 (97Q) fused to mCherry and $400 \mathrm{ng}$ N586 constructs fused to EGFP using Lipofectamine 2000 reagent.

\section{Confocal Imaging}

48 hours after transfection cells were stained with $0.1 \mu \mathrm{g} / \mathrm{mL}$ 2-(4-amidinophenyl)- $1 \mathrm{H}$-indole-6carboxamidine (DAPI) in phosphate buffered saline (PBS) for 15 mins. Then cells were fixed with $4 \%$ (v/v) paraformaldehyde in PBS for $15 \mathrm{~min}$. Cells were imaged on a Leica SP5 confocal microscope using a $40 \times$ or $63 \times$ objective lens. The DAPI channel was collected with an excitation wavelength of $405 \mathrm{~nm}$ and emission wavelengths of 445-500nm; EGFP was collected by excitation at $488 \mathrm{~nm}$ and emission from $520-570 \mathrm{~nm}$; and mCherry was collected by excitation of $561 \mathrm{~nm}$ and emission range of 600-700 nm. Tiled images were taken by using 40× objective lens.

\section{Image analysis}

All microscopic images were processed by Fiji (ImageJ) software [26, 27]. For the quantitation of inclusion types images of cells co-transfected with the different Htt fragment-EGFP fusions and mCherry and then stained with DAPI were analyzed by software Cellprofiler [28] on microscopic images of cells. Cells were automatically detected and traced using a nuclear staining mask (DAPI) and a whole stain mask (mCherry). The settings were calibrated manually by visual inspection for fidelity of object recognition for each experiment (this was not done by a blinded observer). mCherry fluorescence was recorded in each cell and each cell was manually categorized for aggregate phenotype.

\section{Immunofluorescence staining}

COS1 cells were transfected with the human Htt fragment constructs and fixed and processed for immunostaining at $48 \mathrm{~h}$ post-transfection. As a control for ubiquitin staining of cellular aggregates, COS1 cells were treated with $1 \mu \mathrm{M}$ MG132 or equivalent DMSO amount $(0.2 \% \mathrm{v} / \mathrm{v}$ final $)$ for 16 h. For ubiquitin staining, cells were fixed in methanol for $3 \mathrm{~min}$ at $-20{ }^{\circ} \mathrm{C}$ then blocked with $10 \%$ v/v normal goat serum (Thermo Fisher Scientific). For cathepsin D staining, cells were fixed in $4 \%(\mathrm{v} / \mathrm{v})$ paraformaldehyde in PBS for $15 \mathrm{~min}$ at room temperature, permeabilized with $0.2 \% \mathrm{v} / \mathrm{v}$ 
Triton X-100 for $10 \mathrm{~min}$ and then blocked with $10 \% \mathrm{v} / \mathrm{v}$ normal donkey serum (Jackson Immunoresearch). Fixed cells were incubated with mouse anti-ubiquitin (Millipore, mab1510) or goat anti-cathepsin D (Santa Cruz, sc6494) antibodies overnight at $4{ }^{\circ} \mathrm{C}$. Secondary antibodies, donkey anti-goat (Thermo Fischer Scientific) or goat anti-mouse (Jackson Immunoresearch), were conjugated to Alexa Fluor 647. Nuclei were counterstained with $1 \mu \mathrm{g} / \mathrm{ml}$ Hoechst 33342 (Thermo Fisher Scientific) in PBS. Stained cells were imaged on an IN Cell Analyzer 6000 (GE Healthcare) at $40 \times$ magnification using the appropriate excitation/emission channels $(405 \mathrm{~nm} / 455 \mathrm{~nm}, 488$ $\mathrm{nm} / 525 \mathrm{~nm}, 644 \mathrm{~nm} / 706 \mathrm{~nm})$.

\section{Western Blot}

$2.8 \times 10^{5}$ COS 1 cells were plated per well on 12 -well plates. The next day cells were transfected with Lipofectamine 2000 (Invitrogen) reagent containing $1.6 \mu \mathrm{g}$ Htt fragment-EGFP vector. 48 hours after transfection cells were detached and lysed as described previously [29]. Htt fragments-EGFP were dectected using a 1:10000 dilution of an antibody to GFP (A6455: Life Technologies).

\section{Statistics}

For evaluation of differences in means (and S.E.M.) the Kruskal Wallis non-parametric 1 way ANOVA with post-hoc Dunns test was used for samples comparisons using Prism (Graphpad) software packages. Differences with $\mathrm{P}<0.05$ were considered significant $(*), \mathrm{P}<0.01$ were displayed as $(* *)$ and $\mathrm{P}<0.001$ were displayed as $(* * *)$.

\section{RESULTS}

We first constructed an array of eight $\mathrm{N}$-terminal fragment constructs that match some of the more commonly studied fragments fused at the N-terminus of EGFP (Fig. 1A). This included lengths for exon 1, which has 90 amino acids using the 23Q wild-type polyQ length nomenclature (N90), and N1052, N117, N171, N513, N536, N552, and N586. Each construct was made with a 25Q length and a corresponding 97Q length (for a total of 16 constructs). All constructs expressed products of the anticipated mass by Western Blot (Fig. 1B).

As anticipated, all fragments with a HD-associated polyQ length (97Q) formed visible cytoplasmic aggregates in cells when expressed transiently from a CMV promoter (Fig. 2A). Unexpectedly, the longer fragments (above 117 amino acids) with a wild-type polyQ length (25Q) expressed under the same conditions also formed visible foci regardless of the polyQ length, and did so to a similar extent as the 97Q counterpart (Fig. 2A). All constructs had cells with cytoplasmic inclusions except those that expressed N90, N105 and N117 with the 25Q, which did not display any evidence of visible aggregates (Fig. 2A). There was a sharp peak in number of cells with inclusions for 25Q constructs at 171 amino acids. Because aggregation of N90 and other proteins more generally are known to be affected by expression level [30], we measured the comparative expression levels of the fragments by flow cytometry by virtue of the EGFP tag. The expression levels were higher for N90-N105 with 25Q compared to 97Q, whereas expression levels of larger constructs were similar among all constructs regardless of Q length (Fig 2B). One possibility that might exaggerate the appearance of aggregation in cells with the 25Q forms of the fragments is if the 97Q form leads to toxicity and therefore resulted in a selective loss of highest-expressing cells.

$2 \mathrm{~N} x$ Nomenclature for different Htt N-terminal fragments cleaved after the $x$ residue. 
Hence, to test for equivalent gene dosage, a control mCherry construct co-transfected with the Htt fragments. For most fragments there were similar levels of expression, and for the longer fragments (N513-N586), there was more expression of mCherry for the longer 97Q forms, which discounted this possibility being a major factor (Fig 2C). These data hence indicated that the aggregation observed for longer Htt fragments (N171 and longer) was essentially independent of the polyQ expansion (Fig 2B). Another note from this data is that the decreased expression levels of longer Htt fragments (N513-N586) may account for the lower occurrence of cells with aggregates in these populations as the length exceeds N171 (Fig. 2A).

To investigate the non-polyQ mediated aggregation process in more detail, we examined the morphology of the aggregates by confocal microscopy. Across all of the fragment lengths we noticed four morphological categories of visible aggregates (Fig. 3A). These four categories, Types A-C and a mixed (M) group of the other inclusion types were observed in all three different cell types (COS-1, and AD293 and Neuro2a, Fig. 3B) suggesting the three aggregation processes are governed through common mechanisms in different cells. No inclusions were seen in any cell type with fragments less than and including N117 in the 25Q form. Also, N90, N105 and N117 containing 97Q formed only one type of inclusion in all the cell types, which we designated as "Type A" inclusion (Fig. 4). Type A inclusions were characterized by a symmetrical shape, relatively large size (typically $>10 \mu \mathrm{m}$ in diameter) and typical appearance as a singular entity in cells (Fig. 3A). Type A inclusions also correlated to a depletion of "soluble" Htt protein from the rest of the cell (Fig. 5). Type A inclusions only appeared in cells with the pathogenic polyQ expansion (Fig. 5) so their formation seems to be mechanistically driven by the polyQ expansion. Type B aggregates, like Type A were large in size (more than several $\mu \mathrm{m}$ in width) but had a more asymmetric and extended shape with a more variable location in the cytoplasm (Fig. 3A). Cells with type B inclusions appear to retain soluble Htt that had not been sequestered into the inclusion (Fig. 5). Type B aggregates occurred more frequently in cells expressing wild-type polyQ variants and were present in all fragment lengths including and beyond N513. Type C aggregates occurred as an array of multiple small puncta (less than $8 \mu \mathrm{m}$ in width) scattered throughout the cytoplasm (Fig. 3A). The notable feature of this aggregation pattern was the dispersed foci through the cytoplasm (Fig. 5), which are reminiscent of stress granules and/or autophagic vacuoles. Type C aggregates were formed by all Htt lengths including and beyond N171, and were in general more abundant in cells with normal polyQ length (Fig. 4). The fourth category, M appeared to include Type C inclusions concurrently with Type A or B inclusions. The M type occurred in both expanded and unexpanded Q-lengths of fragments longer than and including N536 (Fig. 4).

The dispersed pattern of the Type B and C aggregates appeared similar to previously characterized membrane associated (full length) $\mathrm{Htt}$ in autophagic vacuoles [31, 32]. These vacuoles-Htt structures were previously found to generally lack immunoreactivity to ubiquitin, which is a marker of the inclusions observed in vivo $[2,3]$ and were positive for cathepsin $\mathrm{B}$, which is a marker of lysosomes [31]. Neither Type A, B and C showed immunoreactivity consistent with these prior studies, suggesting the aggregates formed from the fragments are distinct and probably protein-based rather than lipid-associated or autophagic in nature (Fig 6A-C). Specifically, immunostaining of ubiquitin showed Type A inclusions to colozalize peripherally with ubiquitin (Fig 6A). Type B and $\mathrm{C}$ inclusions showed a mild colocalization of ubiquitin staining within the inclusions (Fig 6A). Apart from the rare exception, inclusions for all fragment lengths and polyQ lengths lacked colocalization with cathepsin $\mathrm{D}$ above background levels and mostly appeared to exclude the cathepsin D (Fig 6C). 
We next assessed whether aggregate type was dependent on expression levels of the Htt fragments. Because the expression level of short fragments of less than (and including) N171 was at least 4fold higher than the longer fragments we analyzed these fragments as two separate groups (Shorter fragment set shown in Fig. 7A and the longer set in Fig. 7B). We calibrated the expression levels based on the co-transfected mCherry as a tracer for gene dosage to avoid complications of nonlinearity of the EGFP tag on the Htt fragments as they aggregate. For all fragment lengths cells with aggregates were skewed to higher expression levels, which is consistent with an expression level dependence on aggregation. However, for Type $\mathrm{B}$ and $\mathrm{C}$ inclusions there was no difference in expression level dependence between polyQ lengths for each fragment (except N171; the implications of this exception are discussed further in the discussion), further supporting the independence of these aggregate structures from polyQ-mediated effects. The expression level dependence on Type A was far greater than the other inclusion types, consistent with a polyQmediated aggregation mechanism involving amyloid kinetics.

Because these results suggested Type A inclusions are formed via the expanded polyQ sequence and the others are not, we sought to test whether amyloid kinetics of polyQ could redirect the process of aggregation away from the other inclusion types. For this experiment we co-expressed N90 (in 97Q form) fused to mCherry with N586 in either 25Q or 97Q forms (as EGFP fusions). The N90 inclusions poorly overlapped with the N586 25Q inclusions consistent with them forming through distinct assembly mechanisms (Fig. 8). However, the N586 in 97Q form colocalized extensively around the Type A N90 inclusions. Furthermore, these cells displayed no Type B or C inclusions of N586, which suggested that the polyQ-mediated aggregation kinetically drove aggregation away from the other molecular processes of aggregation upon a template of N90 amyloid structure.

\section{DISCUSSION}

In this study, we described the aggregation process of a range of fragments of Htt that have been used commonly as models of HD pathogenesis. The results have a number of important implications (discussed below in more detail). But before discussion of those points, we stress that we do not imply that these aggregation patterns necessarily reflect the biological mechanisms involving endogenous full length Htt and its proteolysis. Rather we see our results as highlighting the challenges and caveats of using ectopically expressed fragments of Htt to model HD in terms of understanding the relationship between protein aggregation and pathogenesis.

The first key point is that the fragments have inherent information that drives quite distinct patterns of aggregation into visible puncta. Fragments in length up to N117 formed visible aggregates (Type A) strictly in accord with the polyQ expansion in three cell culture models tested (AD293, COS-1 and Neuro2a). However, surprisingly for fragments of N171 and beyond the extent of aggregation was not overly influenced by polyQ expansion suggesting that these longer fragments contain inherently aggregation prone sequences outside the polyQ motif. The extent of aggregation driven by this non-polyQ sequence (at $\sim 10 \%$ of the transfected cell population) was not trivial in that it was similar to the extent of aggregation of other aggregating proteins involved in disease expressed under similar conditions (including superoxide dismutase 1 and polyalanine) $[33,34]$.

As a point of contrast previous studies of N171 containing a short polyQ sequence showed a notably lower level of aggregation than in our study $[35,36]$. The differences may arise from differences in tagging design and expression vectors. For example N171 expressed with a myc 
tag rather than GFP (as we had done) revealed a small fraction of cells with visible aggregates ( $\sim 6$ $\%$ ) for the 25Q length [36]. Also, that study utilized the pcDNA3.1 vector, which expresses several fold lower levels of proteins than the pTIREX vector used in our study even though both drive expression from a CMV promoter (unpublished observations). Kegel et al expressed Htt fused at the N-terminus with a FLAG tag [35]. This study did not report quantitative results on whether this construct aggregated with a short polyQ length (only one cell showing diffuse staining). Hence, it appears that different tags and expression regimes may affect the susceptibility of $\mathrm{Htt}$ fragments to aggregate via non-polyQ mediated mechanisms.

The mechanism of aggregation of the longer fragments (i.e. Type $\mathrm{B}$ and $\mathrm{C}$ aggregates) appears to be driven by a non-amyloid mechanism akin to what we previously described for polyalanine and superoxide dismutase $1[33,34]$. Expanded polyalanine has a strong tendency to assemble into $\alpha$ helical clusters which we previously postulated would cause a derangement of normal oligomeric state as a mechanism of dysfunction [34]. Our work here points to a conceptually similar mechanism (but not necessarily involving $\alpha$-helices) to describe the behavior of the longer $\mathrm{N}$ terminal Htt fragments. This is an important conclusion because it suggests that such aggregates will confer different impacts to cell health than that ascribed to the "gain of toxic" properties for amyloid structures (including amyloidogenic oligomers) [37-39]. This is of most relevance to the study of aggregation in HD models expressing longer proteolytic sequences ectopically, whereby non-polyQ mediated aggregation mechanisms are predicted to dominate the phenotype.

The differential appearance of Type $B$ and $C$ aggregates upon increasing fragment length suggests two different domains drive clustering into these inclusion categories in a competitive manner to the polyQ-driven Type A inclusion. One lies between residues 117 and 171 to dictate Type $\mathrm{C}$ aggregation (Fig 9). This domain could potentially have a normal biological function in fostering Htt engagement into RNA granules, which have a similar morphology to the Type C inclusions [40]. Prior work has suggested that N590 of Htt (in both wild-type and expanded polyQ lengths) can bind RNA in RNA granule subtype P-bodies and participate in regulating mRNA transport in neuron dendrites, which supports this conclusion [41-43]. The first 170 residues of Htt (but not N90) can also interact with other proteins, including $G$ protein-coupled receptor kinase-interacting protein GIT1, which can modulate the aggregation of polyQ-expanded N170 and which may target it to membranous cellular compartments [44]. The other domain driving Type B inclusions lies between residues 171 and 513. A candidate motif for this clustering are the three HEAT repeats, which operate as protein-protein and protein-nucleic acid interaction domains [45, 46]. Prior work has suggested that the HEAT repeat regions in the Htt sequence position of 172-372 can mediate electrostatic interactions with acidic phospholipids, and hence self-association may also involve interactions with lipids [35].

The formation of three competing clustering strategies has a number of implications. The first is that Type B and C clustering mechanisms likely suppress de novo Type A aggregation mechanisms. In vitro, flanking domains can sterically block Htt N90 aggregation into amyloid fibrils, and hence Type B and C clustering may operate similarly [47, 48] (Fig 9). However, once the Type A aggregation mechanism is seeded, the amyloid template provides a dominating reaction kinetic that outcompetes Type B and C clustering mechanisms (as we observed in Fig 8). Without the amyloid template, spontaneous conversion to amyloids occurs at a much lower incidence. Should this mechanism of aggregation be present in the in vivo context, it may influence the spreading progression of Huntington's disease pathology, and invoke a phenotype similar to that proposed for prion propagation of protein aggregate pathology [49]. 
Another implication, if these patterns of aggregation do in fact form from natural cleavage products, is that the appearance of aggregates in pathology may reflect the outcomes of three different clustering processes with different impacts on cell health and survival. This is an important concept because while Htt aggregation into inclusions is a key signature of human pathology, it remains unclear as to whether inclusions form as part of a pathogenic process or alternatively as an adaptive response (or both in different circumstances). In brain, some neuron types accumulate inclusions without apparent loss of survival; whereas other types are lost without obvious inclusion formation (reviewed in [50]). Alternative clustering patterns may involve strategies previously described for differential partitioning of misfolded proteins from amyloid proteins into two distinct foci [51]. The focus comprising misfolded proteins, known as JUxta Nuclear Quality control (JUNQ), is postulated to regulate remedial refolding or degradation of misfolded proteins [51]. The other focus, the Insoluble Protein Deposit (IPOD), operates as a depot for terminally aggregated amyloid proteins including polyQ [51]. Our data is consistent with the aggregation of Type A aggregates falling into the IPOD category and Type B and C falling into the JUNQ category. Hence the multiple inclusion categories could reflect intermediate states or a combinatorial clustering mechanism involving protein self-association and cell-mediated clustering. Hence further knowledge of the how visible aggregates are formed mechanistically is required to properly ascertain the role aggregation plays in pathology.

In conclusion this study has unmasked a complex pattern of self-association of N-terminal Htt fragments driven by two different motifs located between residues 117 and 586. These two types of aggregation are independent of the aggregation caused by polyQ expansion and appear to compete (and indeed largely inhibit) classical polyQ-mediated aggregation. We anticipate these findings to have important ramifications in other studies that relate crude aggregation patterns to the pathogenic process. In particular our results demonstrate the need for a more nuanced assessment of the common models for toxicity involving protein misfolding and aggregation.

\section{ACKNOWLEDGEMENTS}

We would like to thank Steven Finkbeiner from the Gladstone Institute of Neurological Disease for provision of the pGW1-based N90 vectors fused to mCherry. This work was funded by grants to DMH from the Australian Research Council Discovery: Future Fellowships FT120100039, National Health and Medical Research Council Project grants APP1049458, APP1049459 and APP1102059 and the Hereditary Disease Foundation. These funding bodies played no role in the design of the study and collection, analysis, and interpretation of data and in writing the manuscript.

\section{Authors' contributions}

ZC, SAM and ARO designed and performed the experiments and undertook the data analysis, SAM cloned the constructs and prepared the vectors, PJM provided the initial concepts and assisted in preparing the manuscript and DMH oversaw implementation of the experiments, interpretation of the data and co-wrote the manuscript with $\mathrm{ZC}$.

\section{CONFLICT OF INTEREST}

The authors have no conflict of interest to report.

\section{LIST OF ABBREVIATIONS:}

Htt: Huntingtin; polyQ: polyglutamine; HD: Huntington's Disease; Nx: N-terminal Htt fragments up to and including amino acid $x ; \boldsymbol{n Q}$ : polyglutamine sequence containing $n$ glutamines; $\mathbf{C M V}$ : 
Cytomegalus virus; EGFP: Green Fluorescent Protein; M: mixed category of inclusions; FCS: Fetal Calf Serum; DAPI: 2-(4-amidinophenyl)-1H -indole-6-carboxamidine; PBS: Phosphate buffered saline. 


\section{REFERENCES}

[1] MacDonald ME, Ambrose CM, Duyao MP, Myers RH, Lin C, Srinidhi L, et al. A Novel Gene Containing a Trinucleotide Repeat that is Expanded and Unstable on Huntington's Disease Chromosomes. Cell. 1993; 72(6):971-83.

[2] DiFiglia M, Sapp E, Chase KO, Davies SW, Bates GP, Vonsattel JP, et al. Aggregation of huntingtin in neuronal intranuclear inclusions and dystrophic neurites in brain. Science. 1997; 277(5334):1990-3.

[3] Davies SW, Turmaine M, Cozens BA, DiFiglia M, Sharp AH, Ross CA, et al. Formation of Neuronal Intranuclear Inclusions Underlies the Neurological Dysfunction in Mice Transgenic for the HD Mutation. Cell. 1997; 90(3):537-48.

[4] von Hörsten S, Schmitt I, Nguyen HP, Holzmann C, Schmidt T, Walther T, et al. Transgenic rat model of Huntington's disease. Hum Mol Genet. 2003; 12(6):617-24.

[5] Yang S-H, Cheng P-H, Banta H, Piotrowska-Nitsche K, Yang J-J, Cheng ECH, et al. Towards a transgenic model of Huntington/'s disease in a non-human primate. Nature. 2008; 453(7197):921-24.

[6] Wang CE, Tydlacka S, Orr AL, Yang SH, Graham RK, Hayden MR, et al. Accumulation of N-terminal mutant huntingtin in mouse and monkey models implicated as a pathogenic mechanism in Huntington's disease. Hum Mol Genet. 2008; 17(17):2738-51.

[7] Kim YJ, Yi Y, Sapp E, Wang Y, Cuiffo B, Kegel KB, et al. Caspase 3-cleaved Nterminal fragments of wild-type and mutant huntingtin are present in normal and Huntington's disease brains, associate with membranes, and undergo calpain-dependent proteolysis. Proc Natl Acad Sci U S A. 2001; 98(22):12784-9.

[8] Mende-Mueller LM, Toneff T, Hwang SR, Chesselet MF, Hook VY. Tissue-specific proteolysis of Huntingtin (htt) in human brain: evidence of enhanced levels of N- and Cterminal htt fragments in Huntington's disease striatum. J Neurosci. 2001; 21(6):1830-7.

[9] Wellington CL, Ellerby LM, Gutekunst CA, Rogers D, Warby S, Graham RK, et al. Caspase cleavage of mutant huntingtin precedes neurodegeneration in Huntington's disease. J Neurosci. 2002; 22(18):7862-72.

[10] Miller JP, Holcomb J, Al-Ramahi I, de Haro M, Gafni J, Zhang NZ, et al. Matrix Metalloproteinases Are Modifiers of Huntingtin Proteolysis and Toxicity in Huntington's Disease. Neuron. 2010; 67(2):199-212.

[11] Barbaro BA, Lukacsovich T, Agrawal N, Burke J, Bornemann DJ, Purcell JM, et al. Comparative study of naturally occurring huntingtin fragments in Drosophila points to exon 1 as the most pathogenic species in Huntington's disease. Hum Mol Genet. 2015; 24(4):913-25.

[12] Crook ZR, Housman D. Huntington's Disease: Can Mice Lead the Way to Treatment? Neuron. 2011; 69(3):423-35.

[13] Wellington CL, Singaraja R, Ellerby L, Savill J, Roy S, Leavitt B, et al. Inhibiting caspase cleavage of huntingtin reduces toxicity and aggregate formation in neuronal and nonneuronal cells. J Biol Chem. 2000; 275(26):19831-8. 
[14] Landles C, Weiss A, Franklin S, Howland D, Bates G. Caspase-6 does not contribute to the proteolysis of mutant huntingtin in the HdhQ150 knock-in mouse model of Huntington's disease. PLoS Curr. 2012; 4:e4fd085bfc9973.

[15] Goldberg YP, Nicholson DW, Rasper DM, Kalchman MA, Koide HB, Graham RK, et al. Cleavage of huntingtin by apopain, a proapoptotic cysteine protease, is modulated by the polyglutamine tract. Nat Genet. 1996; 13(4):442-49.

[16] Wellington CL, Ellerby LM, Hackam AS, Margolis RL, Trifiro MA, Singaraja R, et al. Caspase cleavage of gene products associated with triplet expansion disorders generates truncated fragments containing the polyglutamine tract. J Biol Chem. 1998; 273(15):9158-67.

[17] Gafni J, Hermel E, Young JE, Wellington CL, Hayden MR, Ellerby LM. Inhibition of calpain cleavage of huntingtin reduces toxicity: accumulation of calpain/caspase fragments in the nucleus. J Biol Chem. 2004; 279(19):20211-20.

[18] Lunkes A, Lindenberg KS, Ben-Haïem L, Weber C, Devys D, Landwehrmeyer GB, et al. Proteases Acting on Mutant Huntingtin Generate Cleaved Products that Differentially Build Up Cytoplasmic and Nuclear Inclusions. Mol Cell. 2002; 10(2):259-69.

[19] Tebbenkamp ATN, Green C, Xu G, Denovan-Wright EM, Rising AC, Fromholt SE, et al. Transgenic mice expressing caspase-6-derived N-terminal fragments of mutant huntingtin develop neurologic abnormalities with predominant cytoplasmic inclusion pathology composed largely of a smaller proteolytic derivative. Hum Mol Genet. 2011; 20(14):2770-82.

[20] Ratovitski T, Gucek M, Jiang H, Chighladze E, Waldron E, D'Ambola J, et al. Mutant Huntingtin N-terminal Fragments of Specific Size Mediate Aggregation and Toxicity in Neuronal Cells. J Biol Chem. 2009; 284(16):10855-67.

[21] Tanaka Y, Igarashi S, Nakamura M, Gafni J, Torcassi C, Schilling G, et al. Progressive phenotype and nuclear accumulation of an amino-terminal cleavage fragment in a transgenic mouse model with inducible expression of full-length mutant huntingtin. Neurobiol Dis. 2006; 21(2):381-91.

[22] Schilling G, Becher MW, Sharp AH, Jinnah HA, Duan K, Kotzuk JA, et al. Intranuclear inclusions and neuritic aggregates in transgenic mice expressing a mutant N-terminal fragment of huntingtin. Hum Mol Genet. 1999; 8(3):397-407.

[23] Hatters DM. Putting huntingtin "aggregation" in view with windows into the cellular milieu. Curr Top Med Chem. 2012; 12(22):2611-22.

[24] Ormsby AR, Ramdzan YM, Mok Y-F, Jovanoski KD, Hatters DM. A Platform to View Huntingtin Exon 1 Aggregation Flux in the Cell Reveals Divergent Influences from Chaperones hsp40 and hsp70. J Biol Chem. 2013; 288(52):37192-203.

[25] Arrasate M, Mitra S, Schweitzer ES, Segal MR, Finkbeiner S. Inclusion Body Formation Reduces Levels of Mutant Huntingtin and the Risk of Neuronal Death. Nature. 2004; 431(7010):805-10.

[26] Schneider CA, Rasband WS, Eliceiri KW. NIH Image to ImageJ: 25 years of image analysis. Nat Methods. 2012; 9(7):671-5. 
[27] Schindelin J, Arganda-Carreras I, Frise E, Kaynig V, Longair M, Pietzsch T, et al. Fiji: an open-source platform for biological-image analysis. Nat Methods. 2012; 9(7):676-82.

[28] Carpenter AE, Jones TR, Lamprecht MR, Clarke C, Kang IH, Friman O, et al. CellProfiler: image analysis software for identifying and quantifying cell phenotypes. Genome Biol. 2006; 7(10):R100.

[29] Olshina MA, Angley LM, Ramdzan YM, Tang J, Bailey MF, Hill AF, et al. Tracking mutant huntingtin aggregation kinetics in cells reveals three major populations that include an invariant oligomer pool. J Biol Chem. 2010; 285(28):21807-16.

[30] Miller J, Arrasate M, Shaby BA, Mitra S, Masliah E, Finkbeiner S. Quantitative Relationships between Huntingtin Levels, Polyglutamine Length, Inclusion Body Formation, and Neuronal Death Provide Novel Insight into Huntington's Disease Molecular Pathogenesis. J Neurosci. 2010; 30(31):10541-50.

[31] Kegel KB, Kim M, Sapp E, McIntyre C, Castano JG, Aronin N, et al. Huntingtin expression stimulates endosomal-lysosomal activity, endosome tubulation, and autophagy. J Neurosci. 2000; 20(19):7268-78.

[32] Velier J, Kim M, Schwarz C, Kim TW, Sapp E, Chase K, et al. Wild-type and mutant huntingtins function in vesicle trafficking in the secretory and endocytic pathways. Exp Neurol. 1998; 152(1):34-40.

[33] Polling S, Mok YF, Ramdzan YM, Turner BJ, Yerbury JJ, Hill AF, et al. Misfolded polyglutamine, polyalanine, and superoxide dismutase 1 aggregate via distinct pathways in the cell. J Biol Chem. 2014; 289(10):6669-80.

[34] Polling S, Ormsby AR, Wood RJ, Lee K, Shoubridge C, Hughes JN, et al. Polyalanine expansions drive a shift into alpha-helical clusters without amyloid-fibril formation. Nat Struct Mol Biol. 2015; 22(12):1008-15.

[35] Kegel KB, Sapp E, Yoder J, Cuiffo B, Sobin L, Kim YJ, et al. Huntingtin associates with acidic phospholipids at the plasma membrane. J Biol Chem. 2005; 280(43):36464-73.

[36] Cooper JK, Schilling G, Peters MF, Herring WJ, Sharp AH, Kaminsky Z, et al. Truncated N-Terminal Fragments of Huntingtin with Expanded Glutamine Repeats form Nuclear and Cytoplasmic Aggregates in Cell Culture. Hum Mol Genet. 1998; 7(5):783-90.

[37] Bucciantini M, Giannoni E, Chiti F, Baroni F, Formigli L, Zurdo J, et al. Inherent Toxicity of Aggregates Implies a Common Mechanism for Protein Misfolding Diseases. Nature. 2002; 416:507-11.

[38] Kayed R, Head E, Thompson JL, McIntire TM, Milton SC, Cotman CW, et al. Common Structure of Soluble Amyloid Oligomers Implies Common Mechanism of Pathogenesis. Science. 2003; 300(5618):486-9.

[39] Laganowsky A, Liu C, Sawaya MR, Whitelegge JP, Park J, Zhao M, et al. Atomic view of a toxic amyloid small oligomer. Science. 2012; 335(6073):1228-31.

[40] Ramdzan YM, Polling S, Chia CP, Ng IH, Ormsby AR, Croft NP, et al. Tracking protein aggregation and mislocalization in cells with flow cytometry. Nat Methods. 2012; 9(5):467-70. 
[41] Ma B, Savas JN, Yu MS, Culver BP, Chao MV, Tanese N. Huntingtin mediates dendritic transport of beta-actin mRNA in rat neurons. Sci Rep. 2011; 1:140.

[42] Savas JN, Makusky A, Ottosen S, Baillat D, Then F, Krainc D, et al. Huntington's disease protein contributes to RNA-mediated gene silencing through association with Argonaute and P bodies. Proc Natl Acad Sc USA. 2008; 105(31):10820-25.

[43] Savas JN, Ma B, Deinhardt K, Culver BP, Restituito S, Wu L, et al. A Role for Huntington Disease Protein in Dendritic RNA Granules. J Biol Chem. 2010; 285(17):13142-53.

[44] Goehler H, Lalowski M, Stelzl U, Waelter S, Stroedicke M, Worm U, et al. A Protein Interaction Network Links GIT1, an Enhancer of Huntingtin Aggregation, to Huntington's Disease. Mol Cell. 2004; 15(6):853-65.

[45] Andrade MA, Perez-Iratxeta C, Ponting CP. Protein Repeats: Structures, Functions, and Evolution. J Struct Biol. 2001; 134(2-3):117-31.

[46] Rubinson EH, Eichman BF. Nucleic acid recognition by tandem helical repeats. Curr Opin Struct Biol. 2012; 22(1):101-09.

[47] Muchowski PJ, Schaffar G, Sittler A, Wanker EE, Hayer-Hartl MK, Hartl FU. Hsp70 and hsp40 chaperones can inhibit self-assembly of polyglutamine proteins into amyloid-like fibrils. Proc Natl Acad Sci U S A. 2000; 97(14):7841-6.

[48] Robertson AL, Bate MA, Buckle AM, Bottomley SP. The Rate of PolyQ-Mediated Aggregation Is Dramatically Affected by the Number and Location of Surrounding Domains. J Mol Biol. 2011; 413(4):879-87.

[49] Brundin P, Melki R, Kopito R. Prion-like transmission of protein aggregates in neurodegenerative diseases. Nat Rev Mol Cell Biol. 2010; 11(4):301-07.

[50] Sieradzan KA, Mann DMA. The selective vulnerability of nerve cells in Huntington's disease. Neuropathol Appl Neurobiol. 2001; 27(1):1-21.

[51] Kaganovich D, Kopito R, Frydman J. Misfolded Proteins Partition Between Two Distinct Quality Control Compartments. Nature. 2008; 454(7208):1088-95.

[52] Harjes P, Wanker EE. The hunt for huntingtin function: interaction partners tell many different stories. Trends Biochem Sci. 2003; 28(8):425-33. 
Fig 1

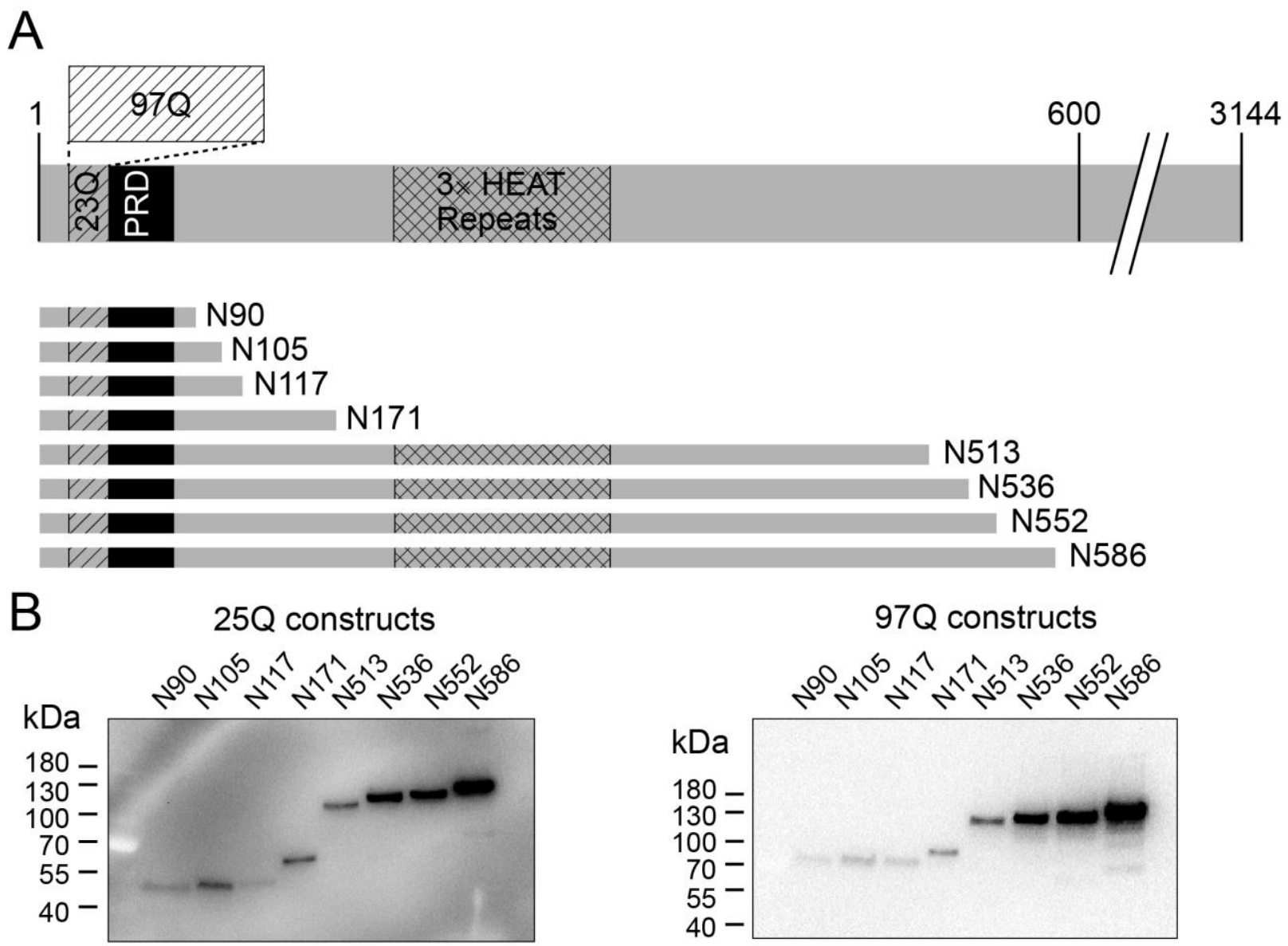

FIGURE 1: Summary of Htt fragment lengths used in this study. A. Shown is a schematic of the amino acid sequence of human Huntingtin (to linear scale) with key domains shown (adapted from [52]). PRD, Proline-rich domain. B. Western Blot of COS1 cells transfected with the constructs from panel A fused to EGFP. Samples were matched approximately to EGFP levels based on GFP fluorescence in lysate. Proteins were probed with GFP antibody. 
Fig 2
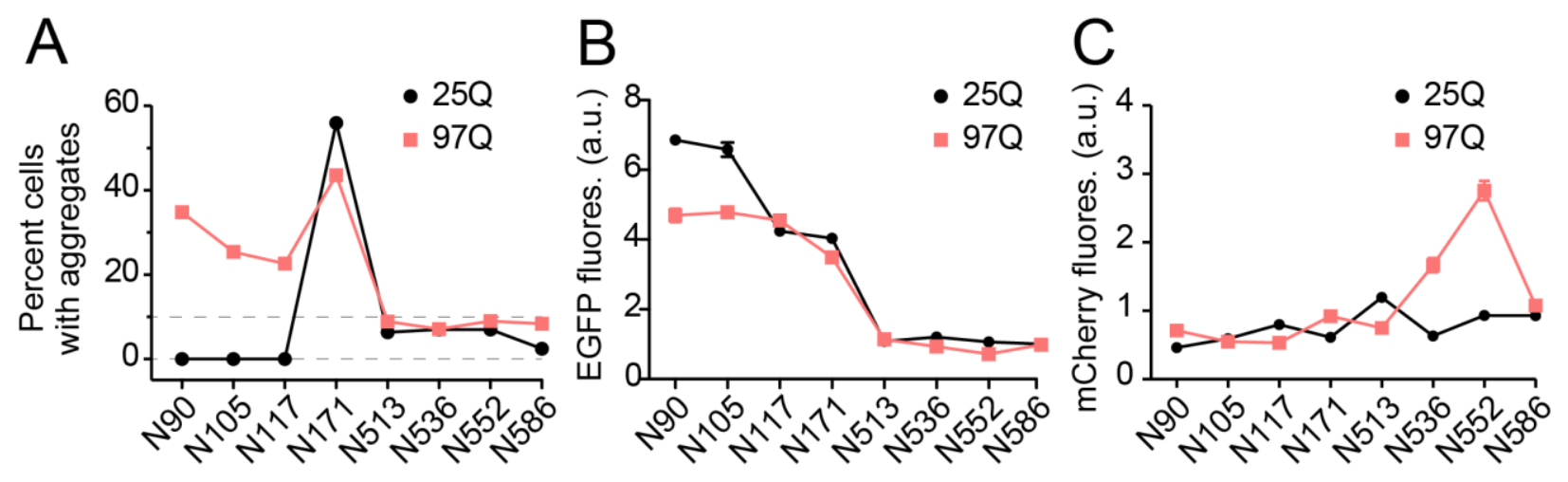

FIGURE 2: Relationship of aggregation profile of $\mathrm{Htt} \mathrm{N}$-terminal fragments with $\mathrm{Htt}$ fragment levels and gene dosage. A. Percent of COS1 cells transfected with the EGFP-tagged fragments that contain visible aggregates measured by microscopy. The dashed gray lines are guides. B. Expression level of Htt fragments based on EGFP fluorescence, measured by flow cytometry. Data shows mean and SEM of median cellular fluorescence $(\mathrm{N}=3$ biological replicates). C. Test of "gene dosage" in transfection using an mCherry tracer co-expressed with the Htt fragments. Data shows mean and SEM of median cellular fluorescence, measured by flow cytometry ( $\mathrm{N}=3$ biological replicates). 


\section{Fig 3}

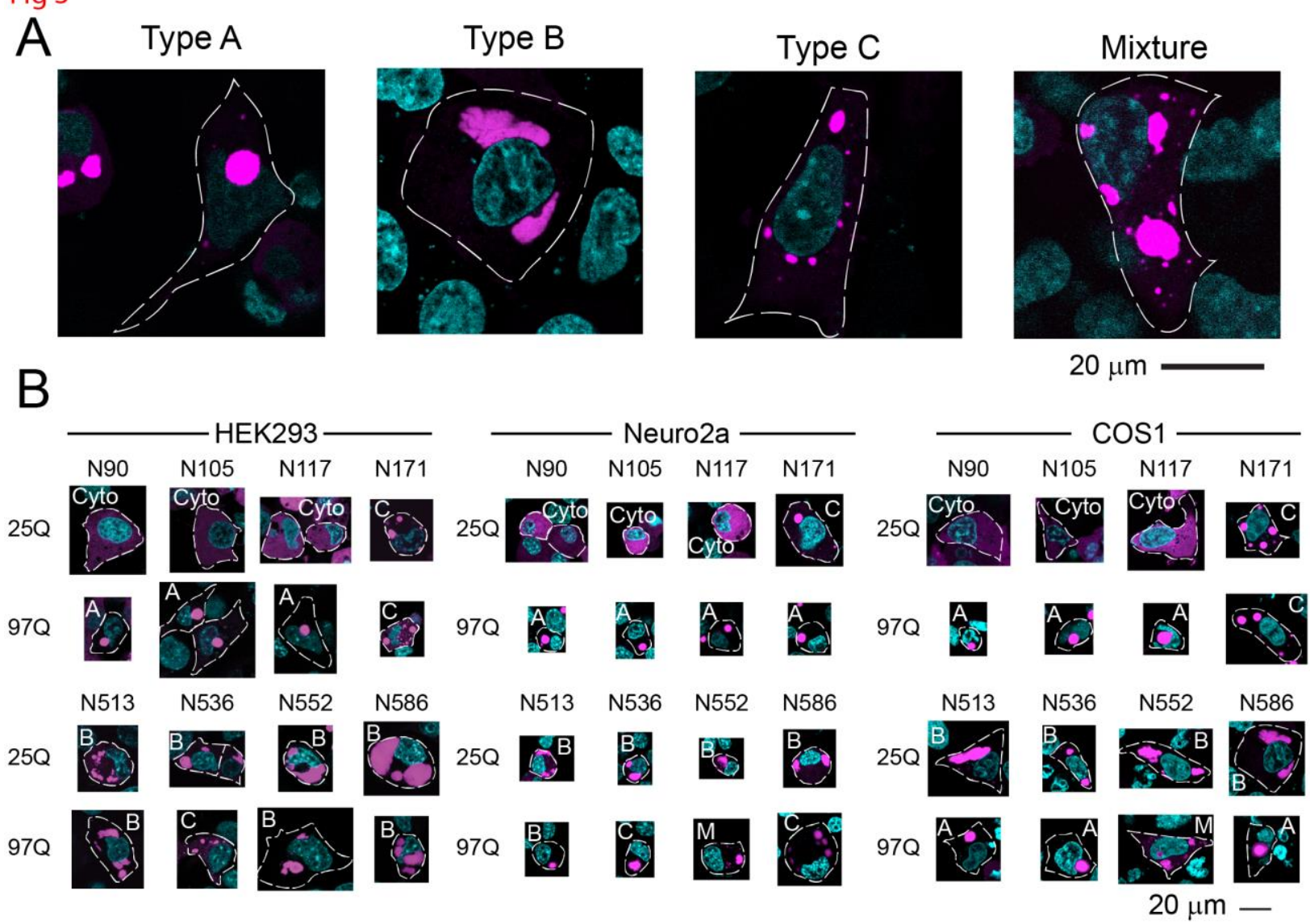

FIGURE 3: Four different inclusion morphologies formed by Htt fragments. A. Shown are representative images of the different categories observed in cultured COS1 cells. Cell outlines indicated by dashed lines based on transmission images. B. The same categories were also seen in Neuro2a and AD293 cells. Representative images shown. 
Fig 4

\section{Type A $\square$ Type B $\square$ Type C $\mathbf{\square}$ Mixture}

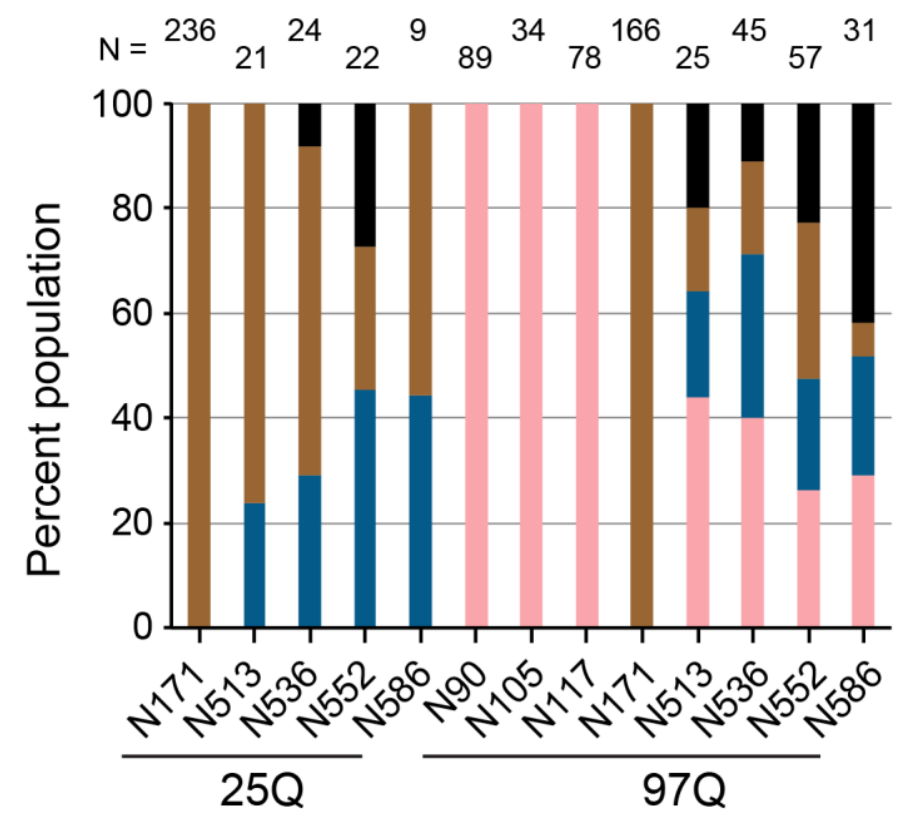

FIGURE 4: The classification of inclusion type in cells containing visible aggregates. Shown are proportion of Types A, B and C in COS1 cells with aggregates at 48 hours post transfection. Note that N90, N105 and N117 in 25Q form does not form visible aggregates. 
Fig 5

Type A

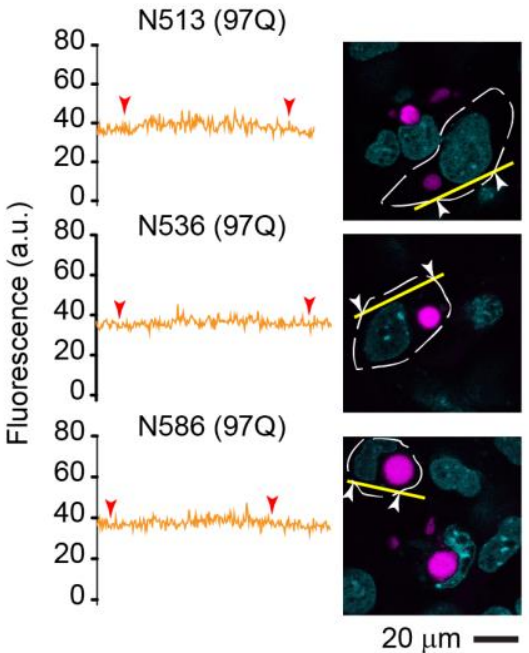

Type B

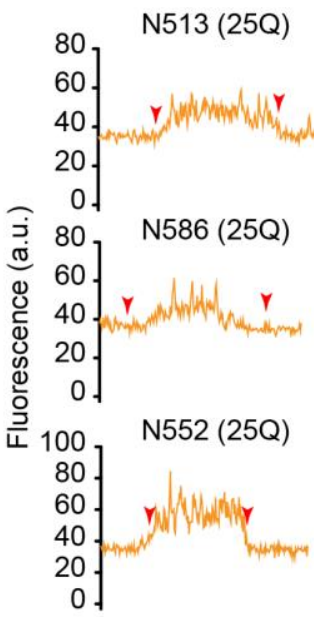

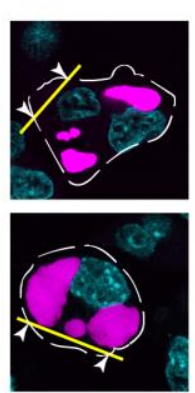

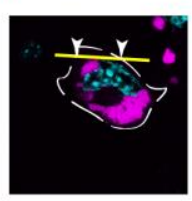

$20 \mu \mathrm{m}-$
Type C
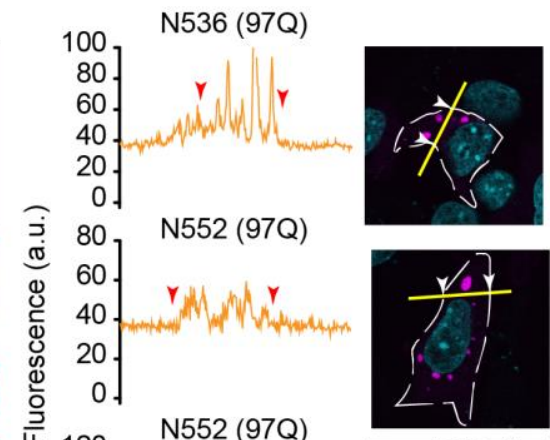

N552 (97Q)

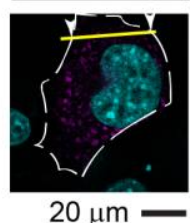

$20 \mu \mathrm{m}$

FIGURE 5. The extent of recruitment of "soluble" Htt fragments into inclusions of different type. Shown are trajectories across the cytoplasm of COS1 cells containing each inclusion category. The trajectories were plotted on the "soluble" pool of Htt and avoided the obvious visible inclusions. The plots show the fluorescence intensity across the trajectory and the edges of the cells are indicated by the arrows. Three representative cells are shown for each category. Cell outlines indicated by dashed lines based on transmission images. 

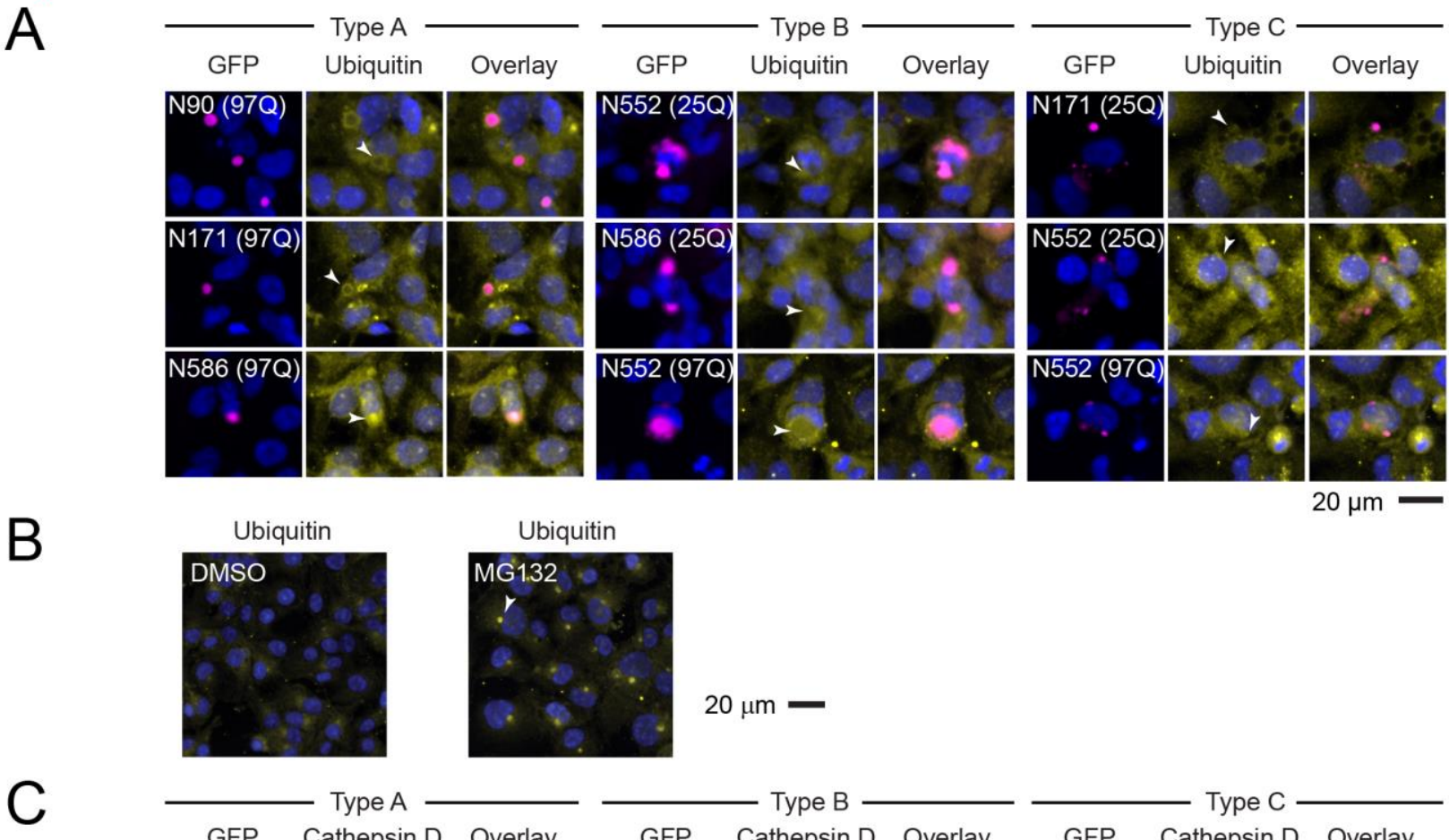

Ubiquitin

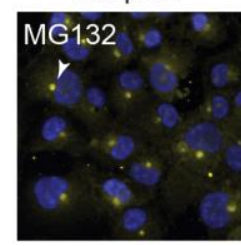

$20 \mu \mathrm{m}-$
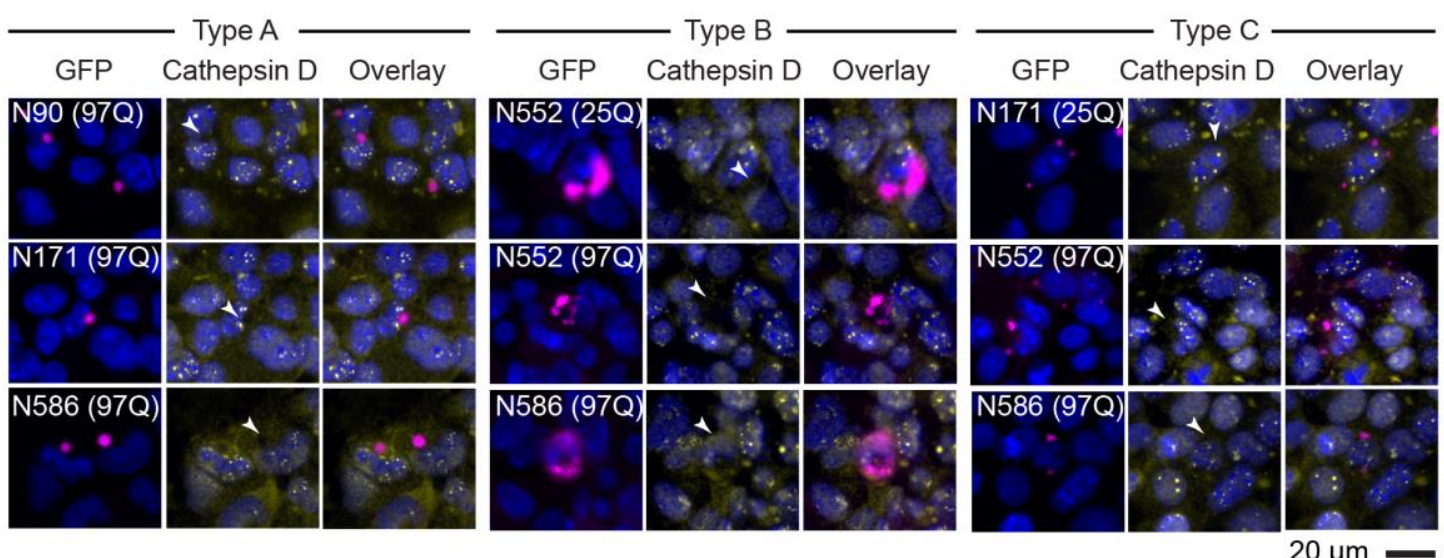

FIGURE 6: Localization of ubiquitin and cathepsin $D$ in relation to $\mathrm{Htt}$ fragment inclusion subtypes. Representative images of fixed cells containing Type A, Type B, or Type C Htt inclusions (pseudocoloured magenta for GFP fluorescence) in COS1 cells at 48 hours post transfection. Cells are counterstained with DAPI (blue) A. Immunostained cells for ubiquitin (pseudocoloured yellow). Arrows indicate position of inclusions. B. Control for ubiquitin staining by inducing protein aggregation with proteasome inhibitor MG132. Cells were treated with $1 \mu \mathrm{M}$ MG132 or the equivalent DMSO control $(0.2 \% \mathrm{v} / \mathrm{v})$ for 16 hours prior to staining. Arrow marks representative ubiquitin focus formed from this treatment. C. Cells stained for cathepsin D (pseudocoloured yellow). Arrows indicate position of Htt-fragment inclusions. 
Fig 7

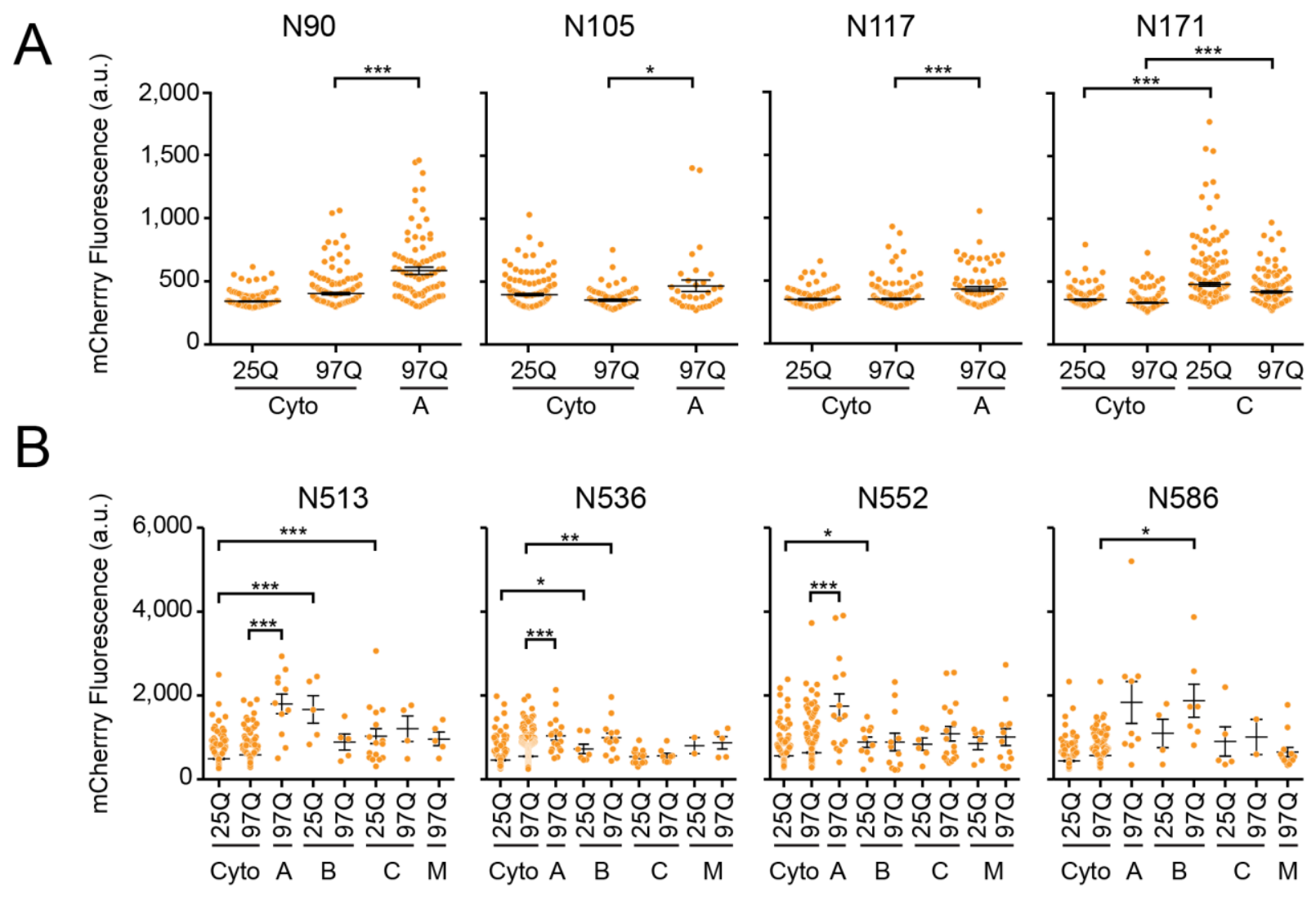

FIGURE 7: Expression level dependence on inclusion subtype. Data shows the mCherry tracer level in COS1 cells as a proxy for gene dosage and transfection level. Each point represents the median cellular mCherry fluorescence. Bars represent means and SEM. A. Shown are the cells with the shorter fragments, with the mCherry fluorescence plotted to the same scale across fragment lengths. B. Shown are the longer fragments, with the mCherry fluorescence plotted to the same scale across fragment lengths but on a different scale to panel A. 
Fig 8

N586 (25Q) N90 (97Q) Overlay
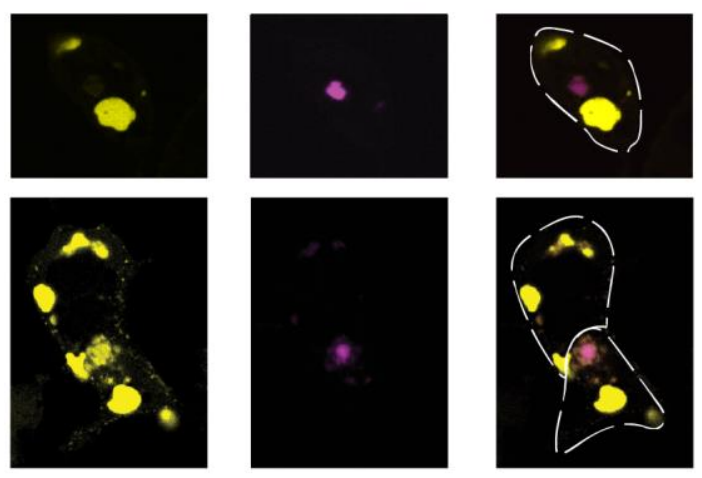

N586 (97Q)
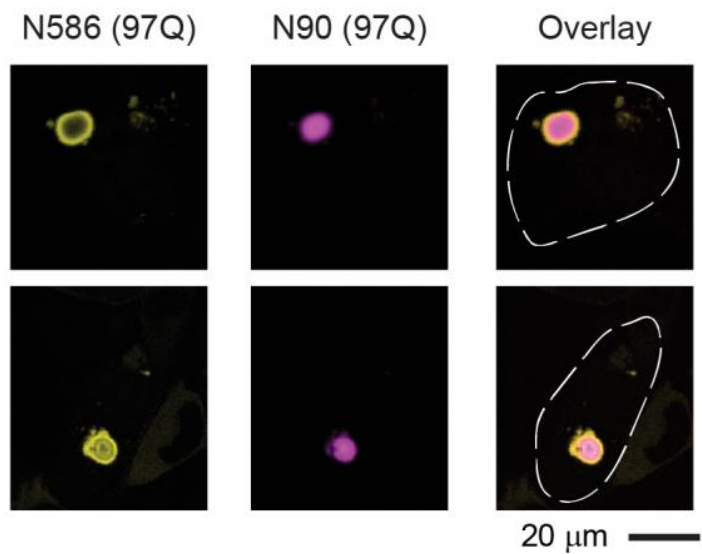

FIGURE 8: Type A inclusions are distinct to Type B but can outcompete with Type B formation for expanded polyQ forms of Htt. Shown are COS1 cells co-transfected with an EGFP tagged N586 fragment and mCherry tagged N90 (97Q) and representative images of cells with inclusions at $48 \mathrm{~h}$ post transfection. Cell outlines indicated by dashed lines based on transmission images. 
Fig 9
- "Type C" aggregation domain between residues 90-171
- "Type B" aggregation domain between residues 171-513
$\square \quad$ HEAT repeats
PolyQ
- Pro-rich domain

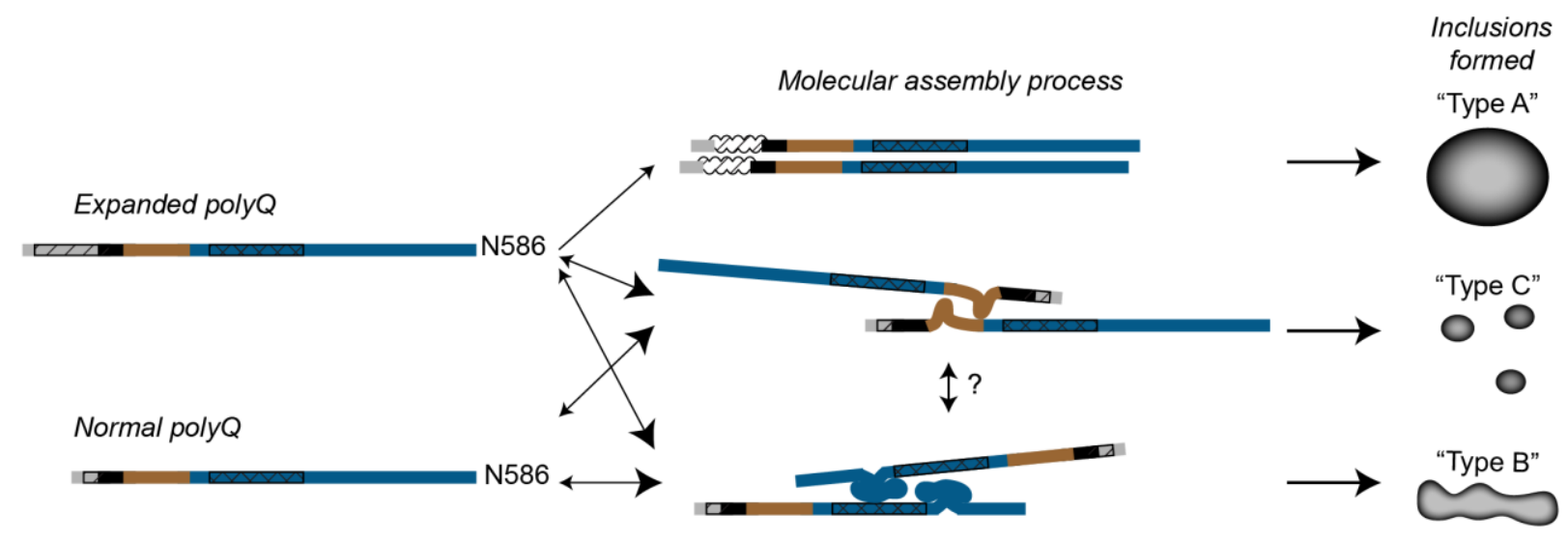

FIGURE 9: Molecular model of how three inclusion states form by Htt N-terminal fragments. The model posits 3 competing clustering mechanisms dictated by two Htt endogenous sequences and the expanded polyQ. 